\title{
Parameters of perineural invasion in radical prostatectomy specimens lack prognostic significance
}

\author{
A David Merrilees ${ }^{1}$, Peter B Bethwaite ${ }^{2}$, Grant L Russell ${ }^{1}$, Richard G Robinson ${ }^{1}$ \\ and Brett Delahunt ${ }^{2}$ \\ ${ }^{1}$ Department of Urology, Wellington Hospital, Wellington, New Zealand and ${ }^{2}$ Department of Pathology \\ and Molecular Medicine, Wellington School of Medicine and Health Sciences, University of Otago, \\ Wellington, New Zealand
}

\begin{abstract}
The prognostic significance of perineural invasion by prostate cancer is debated. We have evaluated the association between biochemical failure and measurements of perineural invasion in radical prostatectomy specimens. Perineural invasion was identified in sections using S-100 protein immunostaining. For nerves showing invasion, the involved nerve closest to the edge of the prostate and to the surgical excision margin, as well as the diameter of these nerves, the largest nerve showing perineural invasion and its proximity to the excision margin, and the percentage of nerves showing perineural invasion up to $1.75 \mathrm{~mm}$ from the excision margin was determined and tested against time to prostate-specific antigen failure, along with preoperative prostate-specific antigen levels, highest Gleason primary grade, Gleason score and TNM T category. Perineural invasion was present in $90 \%$ of cases, with extraprostatic perineural invasion in $25 \%$ of tumors. Diameter of nerves showing perineural invasion ranged from 11 to $680 \mu \mathrm{m}$ and the shortest distance to the surgical excision margin ranged from 33 to $2.57 \mathrm{~mm}$. Perineural invasion density ranged from 6 to $96 \%$. Gleason scores were six in 58 cases, seven in 43 cases, eight in three cases and nine in one case. Clinical T categories were T1c in 75 cases, T2a in 22 cases, T2b in five cases, T2c in two cases, T3 in one case. During a follow-up period of 11 to 55 months (median 26 months), 27 patients showed prostate-specific antigen failure. On univariate analysis only presence of extraprostatic perineural invasion, among parameters of perineural invasion, showed a weak correlation with outcome, while on multivariate analysis this lost significance and preoperative prostatespecific antigen levels, Gleason score and excision margin status were independently associated with biochemical failure. We conclude that the investigated parameters of perineural invasion do not predict prostate-specific antigen recurrence in radical prostatectomy specimens.

Modern Pathology (2008) 21, 1095-1100; doi:10.1038/modpathol.2008.81; published online 23 May 2008
\end{abstract}

Keywords: prostate adenocarcinoma; prostatectomy; perineural invasion; prognosis; outcome

Tumor recurrence or biochemical failure, following attempted curative radical prostatectomy for prostatic adenocarcinoma, is somewhat unpredictable for individual cases. A variety of prognostic parameters have been proposed for these tumors and, in 1999, the College of American Pathologists Consensus Group determined that of these parameters, preoperative serum prostatic-specific antigen (PSA) levels, Gleason score, tumor stage and infiltration of the prostatic capsule by tumor were of proven

Correspondence: Dr B Delahunt, MD, Department of Pathology and Molecular Medicine, Wellington School of Medicine and Health Sciences, University of Otago, Wellington, PO Box 7343, Wellington South 6242, New Zealand.

E-mail: bd@wnmeds.ac.nz

Received 01 February 2008; revised 15 April 2008; accepted 17 April 2008; published online 23 May 2008 prognostic significance. ${ }^{1}$ These features have been incorporated into a proposed prognostic nomogram designed to predict recurrence following curative radical prostatectomy. ${ }^{2}$ The College of American Pathologists Consensus Group further determined that a variety of other proposed prognostic parameters were of unproven clinical value and specifically noted that perineural invasion by tumor required further evaluation as a marker of clinical outcome.

A number of studies have investigated the prognostic utility of perineural invasion in both thin core biopsies $^{3-12}$ and radical prostatectomy specimens ${ }^{13-20}$ with variable results. In the majority of these studies, the absence or presence of perineural invasion was compared with outcome, although, in one study the diameter of the nerve and perineural infiltrate was investigated. 
It has been suggested that the stroma of the perineural sheath promotes tumor growth and that the perineural space acts as a conduit facilitating extraprostatic tumor spread. ${ }^{21}$ In view of this, it would seem intuitive that widespread perineural invasion infiltration of large diameter nerves, and a close proximity of nerves showing perineural invasion to either the prostatic capsule or surgical excision margin may more frequently be associated with biochemical or clinical recurrence and thus have prognostic significance. This study was undertaken to investigate the association between detailed parameters of perineural invasion and biochemical failure in a consecutive series of radical prostatectomy specimens, and to compare these results with those obtained from assessment of established prognostic parameters for prostatic adenocarcinoma.

\section{Materials and methods}

Sections from consecutive radical prostatectomies, performed following histological diagnosis of prostate carcinoma on thin core biopsy in private urological practices in Wellington, New Zealand, between October 1998 and June 2002, were reviewed. The age of the patient at presentation, pretreatment PSA levels and clinical T category were recorded. For each case the whole prostate had been serially sectioned in the gross and all material embedded. Sections were re-examined, and the highest Gleason pattern grade, Gleason score, presence of extraprostatic extension by tumor and excision margin status were determined. In addition, all sections from each case, which contained tumor and included a peripheral zone resection margin were identified. The tissue blocks of these sections were retrieved and fresh sections were then cut and were stained for S-100 protein utilizing routine immunohistochemical methods. Briefly, sections were stained using the streptavidin-biotin method following microwave pretreatment in citrate buffer. Sections were labeled with S-100 protein (Dako, ZO311 Dako Corporation, Carpinteria, CA, USA) followed by biotinylated swine antirabbit (Dako, EO353) and avidin-biotin complex (Dako K0377), Dako Liquid DAB substrate-chromagen system (Dako K3466) with counterstaining by Harris's haematoxylin. S-100 antibody proteinstained sections were examined by light microscopy for evidence of perineural invasion by prostate adenocarcinoma and in those cases where perineural invasion by tumor was identified, the following parameters were determined using a Zeiss Integration eyepiece calibrated for each microscope objective (magnification $\times 2, \times 4, \times 10, \times 20$ and $\times 40$ ); (1) the closest distance to the edge of the prostate of any nerve showing perineural invasion and the cross-sectional diameter of the nerve, (2) the closest distance of any nerve showing perineural invasion to the peripheral zone resection margin (either prostatic or extraprostatic) and the cross-sectional diameter of the nerve, (3) the cross-sectional diameter of the largest nerve showing perineural invasion and the distance of this nerve from the peripheral zone excision margin and (4) perineural invasion density-being the percentage of nerves showing perineural invasion within the superficial peripheral zone (measured from the edge of prostatic capsule to a depth of $1.75 \mathrm{~mm}$ within the prostate).

Patients were followed at two to six monthly intervals and follow-up PSA levels were recorded to detect biochemical failure, which was defined as a persistent elevation in serum PSA levels of $>0.1 \mathrm{ng} / \mathrm{ml}$.

\section{Results}

One hundred and five radical prostatectomies were undertaken in the period under review in patients ranging in age from 47 to 73 years at diagnosis (mean 58 years). Preoperative serum PSA ranged from 0.8 to $52 \mathrm{ng} / \mathrm{ml}$ with a mean of $8.4 \mathrm{ng} / \mathrm{ml}$. The clinical and histological features of the tumors in the series are summarized in Table 1.

The number of sections per case stained for S-100 protein ranged from three to 24 per case (median 12). Perineural invasion by prostatic adenocarcinoma (Figure 1) was identified in 93 cases $(90 \%)$. Perineural invasion was found to be confined to the prostate in 70 cases $(75 \%)$, while in 23 cases $(25 \%)$ there was perineural invasion within extraprostatic tissue. In those cases where tumor was confined within the edge of the prostate, the minimal distance between nerves showing perineural invasion and the prostatic margin for each case ranged from $5 \mu \mathrm{m}$ to $1.66 \mathrm{~mm}$, with the diameter of these nerves ranging from 13 to $350 \mu \mathrm{m}$. The closest distance between nerves showing perineural invasion and the peripheral zone resection margin ranged from $33 \mu \mathrm{m}$ to $2.57 \mathrm{~mm}$ and the cross-sectional diameter of these nerves ranged from 11 to $680 \mu \mathrm{m}$. The crosssectional diameter of the largest nerve showing perineural invasion in each case ranged from 32 to $680 \mu \mathrm{m}$ and the distance of these nerves from the peripheral zone excision margin ranged from $75 \mu \mathrm{m}$ to $3.64 \mathrm{~mm}$. The percentage of nerves showing perineural invasion within $1.75 \mathrm{~mm}$ of the edge of the prostate (perineural invasion density) ranged from six to $96 \%$.

Follow-up data were available for all 105 patients. Follow-up intervals ranged from 11 to 55 months (median, 26 months, mean 28.5 months, s.d. 10.73) and during this period 27 patients demonstrated biochemical relapse, with the relapse interval ranging from 3 to 47 months.

Univariate analyses of clinical and histological parameters showed preoperative serum PSA levels modeled as a continuous variable (Cox coefficient $=0.117, P<0.01)$, highest Gleason pattern grade 
Table 1 Clinical parameters of cases studied

\begin{tabular}{|c|c|c|}
\hline Parameter & $\begin{array}{c}\text { Number of } \\
\text { cases }\end{array}$ & $\begin{array}{c}\text { Cases with } \\
\text { post-operative } \\
\text { biochemical failure }\end{array}$ \\
\hline \multicolumn{3}{|l|}{ TNM T category } \\
\hline T1c & 75 & 17 \\
\hline $\mathrm{T} 2 \mathrm{a}$ & 22 & 9 \\
\hline $\mathrm{T} 2 \mathrm{~b}$ & 5 & 0 \\
\hline $\mathrm{T} 2 \mathrm{c}$ & 2 & 0 \\
\hline T3 & 1 & 1 \\
\hline \multicolumn{3}{|l|}{ Highest Gleason grade } \\
\hline 3 & 58 & 7 \\
\hline 4 & 43 & 19 \\
\hline 5 & 4 & 1 \\
\hline \multicolumn{3}{|l|}{ Gleason pattern grade } \\
\hline $3+3$ & 58 & 7 \\
\hline $3+4$ & 29 & 10 \\
\hline $4+3$ & 14 & 9 \\
\hline $3+5$ & 3 & 0 \\
\hline $4+5$ & 1 & 1 \\
\hline \multicolumn{3}{|l|}{ Gleason score } \\
\hline 6 & 58 & 7 \\
\hline 7 & 43 & 19 \\
\hline 8 & 3 & 0 \\
\hline 9 & 1 & 1 \\
\hline \multicolumn{3}{|l|}{ Surgical margin status } \\
\hline Negative & 79 & 15 \\
\hline Positive & 26 & 12 \\
\hline Extraprostatic extension & 10 & 5 \\
\hline
\end{tabular}

(log-rank $=11.42, P=0.0017$ ), Gleason score (logrank $=16.88, P<0.001$ ) and surgical margin status (log-rank $P=6.74, P=0.007$ ) to be significantly associated with biochemical failure. The association of PSA failure with presence of tumor beyond the edge of the prostate also achieved statistical significance (log-rank $P=3.01, P=0.048$ ), despite the small number of cases in the series that demonstrated this feature.

Of the measured parameters of perineural invasion, division of cases according to the presence or absence of perineural invasion beyond the edge of the prostate showed a weakly significant association $(P=0.047)$ with biochemical failure (Figure 2, Table 2). The presence or absence of perineural invasion within the prostate, the distance of the closest nerve showing perineural invasion to the prostatic capsule or to the surgical excision margin, and the maximum cross-sectional diameter of these nerves, the cross-sectional diameter of the largest nerve showing perineural invasion in each tumor and the proximity of these to the surgical resection margin, and the perineural invasion density all failed to show a significant association with biochemical failure (Table 2). Multivariate analysis, modeling preoperative serum PSA levels, Gleason score, surgical margin status and the presence of

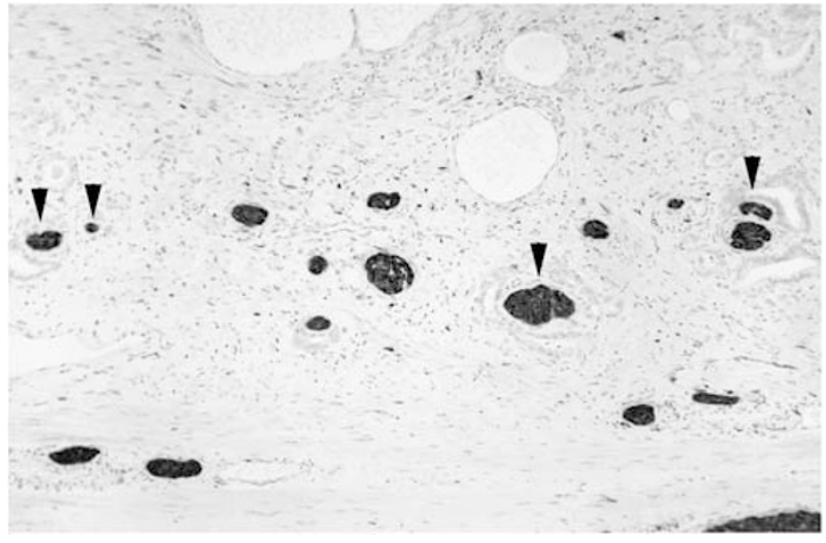

Figure 1 Intraprostatic nerves identified by S-100 protein immunostaining showing perineural infiltration by prostatic adenocarcinoma (arrow).

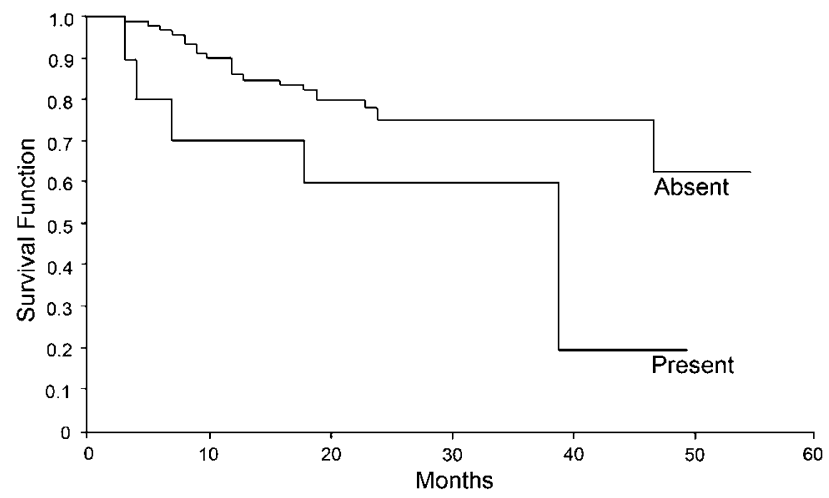

Figure 2 Kaplan-Meier curve for cases divided according to presence or absence of extraprostatic perineural invasion by tumor $(P=0.047)$.

extraprostatic perineural invasion, using Cox's step-down regression, showed only preoperative serum PSA levels, Gleason score and surgical margin status to retain a significant association with postoperative biochemical failure (Table 2).

\section{Discussion}

The prognostic utility of perineural invasion in radical prostatectomy specimens is debated with conflicting results being reported from different series. In particular the presence of perineural invasion has been shown to either correlate ${ }^{13-16,19,20}$ or have no association ${ }^{17,18}$ with biochemical (PSA) recurrence or disease-free survival, following radical prostatectomy on univariate statistical analysis. In those studies where multivariate analysis was undertaken and in which perineural invasion was modeled with various clinical parameters against PSA recurrence, perineural invasion failed to retain significance in analyses that included preoperative serum PSA 
Table 2 Univariate and multivariate analysis of prognostic parameters against biochemical failure

\begin{tabular}{|c|c|c|}
\hline & s.e. & $\begin{array}{l}\text { Significance } \\
\text { (p) }\end{array}$ \\
\hline \multicolumn{3}{|l|}{ Univariate analysis } \\
\hline $\begin{array}{l}\text { 1. Presence/absence } \\
\text { perineural invasion }\end{array}$ & 0.736 & 0.39 \\
\hline $\begin{array}{l}2 \text { i. Closest proximity } \\
\text { of perineural } \\
\text { invasion to prostate } \\
\text { edge }\end{array}$ & 0.675 & 0.43 \\
\hline ii. Diameter of nerve & 3.986 & 0.64 \\
\hline $\begin{array}{l}3 \text { i. Closest proximity } \\
\text { of perineural } \\
\text { invasion to surgical } \\
\text { excision margin }\end{array}$ & 0.769 & 0.16 \\
\hline ii. Diameter of nerve & 3.323 & 0.74 \\
\hline $\begin{array}{l}4 \text { i. Diameter of largest } \\
\text { nerve showing } \\
\text { perineural invasion }\end{array}$ & 3.314 & 0.55 \\
\hline $\begin{array}{l}\text { ii. Distance of nerve } \\
\text { from excision } \\
\text { margin }\end{array}$ & 0.408 & 0.81 \\
\hline $\begin{array}{l}\text { 5. Perineural invasion } \\
\text { density }\end{array}$ & 0.776 & 0.46 \\
\hline $\begin{array}{l}\text { 6. Extraprostatic } \\
\text { perineural invasion }\end{array}$ & 0.370 & 0.047 \\
\hline \multicolumn{3}{|l|}{ Multivariate analysis } \\
\hline - Reoperative PSA & 0.033 & 0.04 \\
\hline - Gleason score & 0.473 & 0.002 \\
\hline $\begin{array}{l}\text { - Excision margin } \\
\text { status }\end{array}$ & 0.459 & 0.04 \\
\hline - T category & 0.453 & 0.99 \\
\hline $\begin{array}{l}\text { - Extraprostatic } \\
\text { perineural invasion }\end{array}$ & 0.653 & 0.59 \\
\hline
\end{tabular}

level, stage, Gleason score and/or the presence of lymph node metastases. ${ }^{13-15,20}$ Despite this one study has shown perineural invasion to significantly correlate with time to biochemical recurrence and this retained significance, along with the presence of lymph node metastases and positive surgical margins, on multivariate testing. ${ }^{16}$ The presence of perineural invasion has also been shown to have a greater positive-predictive value for PSA recurrence than either Gleason score $\geq 7$ and preoperative serum PSA $>10 \mathrm{ng} / \mathrm{ml}^{22}$ These findings, however, are contradicted by the rather surprising observation that the presence of perineural invasion was associated with increased biochemical recurrence-free survival interval. ${ }^{19}$

While perineural invasion is recognized as a common feature of prostate adenocarcinoma in glands removed by radical prostatectomy, the proportion of involved nerves varies from series to series. In studies based upon a review of pathology reports, without re-examination of histology, perineural invasion was noted in $31.9-60.5 \%$ of specimens, ${ }^{16,22,23}$ while in series in which review of sections was undertaken, the incidence ranged from 74 to $84 \%$, with the majority series being in the order of $75 \% \cdot{ }^{13-15,17-19}$ Our finding that $90 \%$ of specimens showed perineural invasion, following identification of nerves using S-100 protein immunostaining, demonstrates that even after review, some nerves showing perineural invasion are overlooked. It is of interest that the two series that showed perineural invasion to have a significant association with biochemical recurrence on multivariate analysis were based on examination of histology reporting without formal review of the sections by a pathologist. ${ }^{16,22}$ This, along with small sample size of some series, may have acted as a confounding influence in previous studies that have investigated the prognostic significance of perineural invasion in radical prostatectomy specimens.

Of the parameters of perineural invasion that we studied, the presence of perineural invasion beyond the edge of the prostate showed a weak correlation with biochemical failure $(P=0.047)$, which was lost on multivariate analysis. Extraprostatic extension by tumor is recognized as having an association with tumor recurrence, being the defining feature of the pT3a category of the AJCC staging system for prostatic adenocarcinoma. ${ }^{24}$ The prognostic significance of this parameter has been recently confirmed and it has been further shown that radial distance of extraprostatic spread correlates with biochemical failure. ${ }^{25}$ Our finding of a weak association between extraprostatic perineural invasion and biochemical failure may simply be a reflection of the poor prognosis associated with extraprostatic tumor spread. The observation that this parameter failed to retain prognostic significance in a multivariate analysis that included other parameters of tumor spread suggests that the perineural location of extraprostatic tumor deposits does not itself facilitate tumor dissemination.

In an attempt to improve the prognostic utility of perineural invasion in radical prostatectomy specimens, Maru et al, ${ }^{15}$ stratified tumors showing perineural invasion according to the cross-sectioned diameter of the largest involved nerve. They demonstrated that, while the presence or absence of perineural invasion was not significantly associated with tumor progression on multivariate analysis, diameter of involved nerves was associated with progression. When cases were grouped at intervals of $0.25 \mathrm{~mm}$ according to largest nerve diameter, increasing diameter correlated with higher pathologic stage, increasing tumor volume, and for nerves showing perineural invasion tumors within crosssectional diameters 0.25 to $<0.50 \mathrm{~mm}, 0.50$ to $<0.75 \mathrm{~mm}$ and $\geq 0.75 \mathrm{~mm}$, increasing likelihood of biochemical recurrence. In that study, the perineural invasion diameter was taken as the sum of the nerve diameter and the diameter of investing tumor. As such, high perineural invasion diameters could represent either a small volume tumor infiltrating a large diameter nerve or extensive tumor growth in and around the perineural sheath of a small nerve. 
We attempted to quantify the tumor volume of each involved nerve but found difficulties associated with reproducibility due to frequent eccentric distribution of tumor deposits. Further, there was considerable variation in the volume of tumor at different points along individual nerves (data not shown) and we considered that this had the potential to introduce an unacceptable level of sampling error into the assessment. Because of these difficulties we confined our measurement to the diameter of the involved nerve, rather than a combined diameter of tumor and nerve, and have demonstrated that this shows no correlation with biochemical recurrence when modeled as a continuous variable. This would suggest that the observed correlation between perineural invasion diameter and tumor progression as determined by Maru et $a l,{ }^{15}$ is dependent on the volume of the perineural tumor infiltrate, which in turn, may simply reflect tumor growth rate.

It has been demonstrated, in both in vitro models and in human tissue samples that, following invasion into the perineural space, tumor cells show increased proliferative activity and a decreased apoptotic rate. ${ }^{26}$ It is also recognized that proximity of intraprostatic tumor to the resection margin in radical prostatectomy specimens is not of prognostic significance. ${ }^{27}$ In view of this, our finding that the presence of proximity of perineural invasion to the prostatic capsule or excision margin is not associated with a higher rate of biochemical failure implies that, while infiltration of the perineural space may promote local tumor growth, it does not necessarily facilitate extraprostatic spread.

In conclusion this study has shown that parameters of perineural invasion are not of prognostic significance, as determined by PSA recurrence, for patients with prostate adenocarcinoma who have undergone radical prostatectomy.

\section{References}

1 Bostwick DG, Grignon DJ, Hammond EH, et al. Prognostic factors in prostate cancer. College of American Pathologists consensus statement 1999. Arch Pathol Lab Med 2000;124:995-1000.

2 Ohori M, Kattan M, Scardino PT, et al. Radical prostatectomy for carcinoma of the prostate. Mod Pathol 2004;17:349-359.

3 Egan AJ, Bostwick DG. Prediction of extraprostatic extension of prostate cancer based on needle biopsy findings: perineural invasion lacks significance on multivariate analysis. Am J Surg Pathol 1997;21: 1496-1500.

4 Stone NN, Stock RG, Parikh D, et al. Perineural invasion and seminal vesicle involvement predict pelvic lymph node metastasis in men with localized carcinoma of the prostate. J Urol 1998;160:1722-1726.

5 Vargas SO, Jiroutek M, Welch WR, et al. Perineural invasion in prostate needle biopsy specimens. Correla- tion with extraprostatic extension at resection. Am J Clin Pathol 1999;111:223-228.

6 de la Taille A, Katz A, Bagiella E, et al. Perineural invasion on prostate needle biopsy: an independent predictor of final pathologic stage. Urology 1999;54: 1039-1043.

7 Cheng L, Slezak J, Bergstralh EJ, et al. Preoperative prediction of surgical margin status in patients with prostate cancer treated by radical prostatectomy. J Clin Oncol 2000;18:2862-2868.

8 D’Amico AV, Wu Y, Chen M-H, et al. Perineural invasion as a predictor of biochemical outcome following radical prostatectomy for select men with clinically localized prostate cancer. J Urol 2001;165: 126-129.

9 Sebo TJ, Cheville JC, Riehle DL, et al. Perineural invasion and MIB-1 positivity in addition to Gleason score are significant preoperative predictors of progression after radical retropubic prostatectomy for prostate cancer. Am J Surg Pathol 2002; 26:431-439.

10 O’Malley KJ, Pound CR, Walsh PC, et al. Influence of biopsy perineural invasion on long-term biochemical disease-free survival after radical prostatectomy. Urology 2002;59:85-90.

11 Quinn DI, Henshall SM, Brenner PC, et al. Prognostic significance of preoperative factors in localized prostate carcinoma treated with radical prostatectomy. Cancer 2003;97:1884-1893.

12 Cannon GM, Pound CR, Landsittel DP, et al. Perineural invasion in prostate cancer biopsies is not associated with higher rates of positive surgical margins. Prostate 2005;63:336-340.

13 Ravery V, Boccon-Gibod LA, Meulemans A, et al. Predictive value of pathological features for progression after radical prostatectomy. Europ Urol 1994;26: 197-201.

14 van den Ouden D, Hop WC, Kranse R, et al. Tumor control according to pathological variables in patients treated by radical prostatectomy for clinical localized carcinoma of the prostate. Br J Urol 1997; 97:203-211.

15 Maru N, Ohori M, Kattan MW, et al. Prognostic significance of the diameter of perineural invasion in radical prostatectomy specimens. Hum Pathol 2001;32: 828-833.

16 Ozcan F. Correlation of perineural invasion on radical prostatectomy specimens with other pathologic prognostic factors and PSA failure. Europ Urol 2001; 40:308-312.

17 Ito K, Nakashima J, Mukai M, et al. Prognostic implication of microvascular invasion in biochemical failure in patients treated with radical prostatectomy. Urol Int 2003;70:297-302.

18 Ng JC, Koch MO, Daggy JK, et al. Perineural invasion in radical prostatectomy specimens: Lack of prognostic significance. J Urol 2004;172:2249-2251.

19 Ramsden AR, Chodak G. An analysis of risk factors for biochemical progression in patients with seminal vesicle invasion: Validation of Kattan's nomogram in a pathologic subgroup. BJU Int 2004;93: 961-964.

20 Miyake H, Sakai I, Harada K, et al. Limited value of perineural invasion in radical prostatectomy specimens as a predictor of biochemical recurrence in Japanese men with clinically localized prostate cancer. Acta Urol Japan 2005;51:241-246. 
21 Cornell RJ, Rowley D, Wheeler T, et al. Neuroepithelial interactions in prostate cancer are enhanced in the presence of prostatic storma. Urology 2003;61: 870-875.

22 Endrizzi J, Seay T. The relationship between early biochemical failure and perineural invasion in pathological T2 prostate cancer. BJU Int 2000;85:696-698.

23 Shariat SF, Khoddami SD, Saboorian H, et al. Lymphovascular invasion is a pathological feature of biologically aggressive disease in patients treated with radical prostatectomy. J Urol 2004;171:1122-1127.

24 Greene FL, Page DL, Fleming ID. AJCC Cancer Staging Manual. Springer-Verlag: New York, 2002.
25 Sung M-T, Lin H, Koch MO, et al. Radial distance of extraprostatic extension measured by ocular micrometer is an independent predictor of prostate-specific antigen recurrence: a new proposal for the substaging of pT3a prostate cancer. Am J Surg Pathol 2007;31:311-318.

26 Ayala GE, Dai H, Ittmann $M$, et al. Growth and survival mechanisms associated with perineural invasion in prostate cancer. Cancer Res 2004;64:6082-6090.

27 Emerson RE, Koch MO, Daggy JK, et al. Closest distance between tumor and resection margin in radical prostatectomy specimens. Lack of prognostic significance. Am J Surg Pathol 2005;29:225-229. 